Krisztina Molnár

Pécs

\title{
Das Infinitivsubjekt in Kausativkonstruktionen im Sprachvergleich
}

DOI: 10.14232/fest.bassola.5

\begin{abstract}
Dass das logische Subjekt des Infinitivs bei Kausativkonstruktionen über den Akkusativ hinaus auch mit einer Präpositionalphrase ausgedrückt werden bzw. fehlen kann, ist hinlänglich bekannt. Da die Präpositionen, die in solchen Fällen auftreten, dieselben sind wie bei Agensausdrücken in Passivsätzen, werden Konstruktionen dieses Typs als „passivähnlich“ oder als „Kausativpassiv“ bezeichnet. Unklar ist jedoch, ob dieses Kausativpassiv als eine Art passivische Diathese zu kanonischen Kausativkonstruktionen betrachtet werden kann. Im vorliegenden Beitrag werden Ergebnisse einer Pilotanalyse beschrieben, in der semantische Eigenschaften von Infinitivsubjekten in kanonischen Kausativkonstruktionen und im Kausativpassiv in sprachvergleichender Perspektive untersucht wurden. Die Ergebnisse legen nahe, dass das Kausativpassiv unter den Vergleichssprachen lediglich im Italienischen eine mögliche Passivdiathese darstellt.
\end{abstract}

\section{Einleitung}

Kausation ist ein viel untersuchtes Phänomen in den Sprachen der Welt. Kausativkonstruktionen drücken, vereinfacht gesagt, Veranlassen (1) oder Zulassen (2) aus und werden im Deutschen in der Regel als AcI-Konstruktionen realisiert:

(1) DT. Der Krimiautor lässt den Detektiv einen schweren Fall lösen.

(2) DT. Die Eltern lassen ihren Sohn bei seinem Schulfreund übernachten.

Ein wichtiges Charakteristikum von AcI-Konstruktionen ist, dass sie neben einem Matrixverb (hier: lassen) und einem Infinitiv auch ein akkusativisches Glied enthalten, das gleichzeitig als das logische Subjekt des Infinitivs fungiert. 
Dieses Infinitivsubjekt kann aber auch durch eine Präpositionalphrase ausgedrückt werden (3) oder gänzlich fehlen (4):

(3) DT. Zurzeit lässt der Verwaltungsrat die Situation von externen Experten analysieren. (A09/JAN.00001 St. Galler Tagbl., 03.01.2009, S. 31)

(4) DT. Sie wollen in vier Jahren 45 Millionen Bäume pflanzen lassen [...]. (B08/JAN.00883 Berliner Ztg., 04.01.2008 [S. 8])

Solche Konstruktionen (in erster Linie die mit einem präpositionalen Infinitivsubjekt) werden in der Literatur häufig als „passivähnlich“ (Zifonun et al. 1997: 1415), „semipassiv“ (Hyvärinen 1989: 167) oder als „Kausativpassiv“ (Gunkel 2003: 61) bezeichnet, die weiteren Eigenschaften dieser Konstruktionen sind aber weitgehend unerforscht.

Diese Formvarianz bei den Infinitivsubjekten ist kein Alleinstellungsmerkmal des Deutschen, da sich Vergleichbares auch bei Kausativverben des Italienischen und bei permissiven Verben des Ungarischen beobachten lässt.

Beim vorliegenden Beitrag handelt es sich um einen Versuch, mehr über das sog. Kausativpassiv zu erfahren, indem die semantischen Eigenschaften von Infinitivsubjekten erfasst werden. Dabei werden die kanonischen Kausativkonstruktionen (im Weiteren KK; mit einem akkusativischen Infinitivsubjekt) und das Kausativpassiv (im Weiteren KP; mit einem präpositional ausgedrückten Infinitivsubjekt) separat behandelt, damit die eventuellen Unterschiede zwischen den beiden Konstruktionstypen aufgedeckt werden.

Als Datengrundlage für die Pilotanalyse dienen Belege aus elektronisch verfügbaren Korpora der Vergleichssprachen (DeReKo, COLFIS, PAISÀ, MNSZ).

Im Folgenden werden zuerst die Realisierungsmöglichkeiten von Infinitivsubjekten in Kausativ- und Permissivkonstruktionen in den Vergleichssprachen Deutsch, Italienisch und Ungarisch kurz skizziert. In Abschn. 3 werden dann die Ergebnisse einer Pilotanalyse dargestellt, in der semantische Eigenschaften von Infinitivsubjekten in kanonischen KK und im KP untersucht wurden. Zum Schluss werden die Ergebnisse zusammengefasst und weitere Forschungsfragen formuliert. 


\section{Form des Infinitivsubjekts}

Kausativ- und Permissivkonstruktionen werden in unseren Vergleichssprachen in der Regel als AcI-Konstruktionen realisiert ${ }^{1}$ (vgl. Molnár 2018: 87ff.), so erscheint das Infinitivsubjekt typischerweise als eine NP im Akkusativ:

(5) DT. Die Marine lässt 820 Wehrpflichtige erst im Februar antreten. (DPA09/JAN.00634 dpa, 02.01.2009)

Das Infinitivsubjekt kann aber auch gänzlich fehlen (6), in solchen Fällen ist der Referent des Infinitivsubjekts unbekannt oder unwichtig (vergleichbar etwa mit der Bedeutung von man):

(6) DT. Spanien ließ daraufhin die Grenzbefestigungen ausbauen und die Zäune auf bis zu sechs Meter erhöhen. (DPA09/JAN.00236 dpa, 01.01.2009)

Des Weiteren kann das Infinitivsubjekt auch durch eine Präpositional- oder Postpositionalphrase ausgedrückt werden:

(7) Dт. Schibli und Kunz kritisieren, dass der Vorstand die Parole nicht von der Delegiertenversammlung des Bauernverbandes absegnen liess. (A09/JAN.02005 St. Galler Tagbl., 10.01.2009, S. 5)

$\begin{array}{llllll}\text { (8) IT. } & \mathrm{Si} & \text { fece } & \text { ritrarre } & \text { da } & \text { Tiziano [...]. (PAISÀ) } \\ & \text { sich } & \text { mach.REM.3SG } & \text { porträtieren } & \text { PRÄP } & \text { Tizian }\end{array}$ „Er ließ sich von Tizian porträtieren.

(9) UNG. nem hagyja magát az ellenség által irányítani. $\begin{array}{llll} & & & \text { (MNSZ) } \\ \text { NEG lass.3Sg sich DEF.ART Feind postP lenken }\end{array}$ ,Er lässt sich nicht vom Feind lenken.`

\footnotetext{
Im Ungarischen bezieht sich diese Behauptung nur auf die Permissivkonstruktionen, da Kausation morphologisch ausgedrückt wird.

2 In den Glossen werden folgende Abkürzungen verwendet: IMP: imperfetto, REM: passato remoto, PST: Vergangenheit, FUT: Futur, DEF.ART: definiter Artikel, PRÄP: Präposition, PRÄP.DEF: Präposition mit dem definiten Artikel, PostP: Postposition, NEG: Negation, sG: Singular, PL: Plural, 3: 3. Person.
} 
$\mathrm{Zu}$ beachten ist hierbei, dass in solchen Fällen das Infinitivsubjekt formal identisch ist mit den Agensausdrücken in Passivkonstruktionen (selbst im Ungarischen, wo das morphologische Passiv nicht mehr verwendet wird). Das Verb (bzw. der Infinitiv) selbst zeigt jedoch keine Passivmorphologie (vgl. auch Molnár 2018: 110ff.; Serianni 1991: 550f.; Zifonun et al. 1997: 1415).

\section{Analyse}

Im Folgenden werden semantische Eigenschaften von Infinitivsubjekten in kanonischen KK sowie im KP in den drei Vergleichssprachen untersucht. Die Pilotanalyse soll einerseits zeigen, ob die Konstruktionstypen bzw. die Vergleichssprachen Unterschiede in dieser Hinsicht aufweisen. Andererseits sollen weitere Richtungen in der Beschreibung des KP aufgedeckt werden.

In die vorliegende Pilotanalyse wurden für jedes Matrixverb jeweils 200 nach dem Zufallsprinzip ausgewählte Belege mit kanonischen KK und mit dem KP aufgenommen. Die untersuchten Matrixverben sind lassen, fare (,machen'), lasciare (,lassen') und hagy (,lassen', ,zulassen'). Von diesen Verben können lassen und fare sowohl eine kausative als auch eine permissive Lesart haben, die beiden anderen Verben verfügen nur über eine permissive Lesart.

In den Vergleichssprachen ist das Subjekt in der Regel auch das Agens (10), es sind aber auch Subjekte möglich, die eine andere thematische Rolle, wie z.B. Patiens (11) oder Experiencer (12) haben:

(10) DT. Petra kocht die Suppe.

(11) DT. Die Suppe kocht.

(12) DT. Petra sieht Hans.

Problematisch ist jedoch, dass die semantischen Rollen keine distinkten Kategorien sind (vgl. z. B. Dowty 1991: 571), so ist die Zuweisung von semantischen Rollen nicht immer eindeutig. ${ }^{3}$

\footnotetext{
Die Literatur zu semantischen Rollen ist abundant. Besonders viel diskutiert wurden die beiden wichtigsten Rollen „Agens“ und „Patiens“ (vgl. z.B. Dowty 1991; Foley / Van Valin 1984). Hier kann eine Auseinandersetzung mit der Literatur nicht angestrebt werden. Für die vorliegende Arbeit war nur wichtig, semantische Merkmale von Infinitivsubjekten zu identifizieren, mit denen im Laufe der Pilotanalyse gearbeitet werden konnte.
} 
Für die vorliegende Untersuchung hat die semantische Rolle des Agens eine besondere Relevanz. Das Agens ist typischerweise eine Person, die absichtlich handelt und das durch das Prädikat beschriebene Geschehen verursacht und kontrolliert. Eine weitere wichtige Eigenschaft des Agens ist die Volitionalität, d. h. dass es freiwillig handelt (vgl. Duden 2009: 390; Primus 2012: 16f.). In Kausativkonstruktionen wird die Frage nach dem Agens dadurch verkompliziert, dass es einen Kausator/Verursacher gibt, der jemanden zu einer Handlung veranlasst. Der Kausatorausdruck fungiert als das grammatische Subjekt des Satzes, der Referent ist aber nicht identisch mit der Person, welche die Handlung ausführt:

(13) DT. Der Lehrer lässt den Schüler das Gedicht vorlesen. gramm. Subj. logisches Subj. von vorlesen

Kausator Agens

Somit ist das Agens (als handelnde Person) nicht identisch mit dem grammatischen Subjekt des Satzes. Gleichzeitig büßt es eine wichtige Funktion ein: Von Volitionalität kann in diesen Fällen kaum gesprochen werden.

\subsection{Kanonische Kausativkonstruktionen}

Bei den kanonischen KK bin ich von der Annahme ausgegangen, dass als Infinitivsubjekte in erster Linie Ausdrücke fungieren, die stark agentivisch sind, d.h. Phrasen, die Personen bezeichnen, die die von ihnen durchgeführte Handlung kontrollieren. Die Hypothese konnte jedoch nicht bestätigt werden, wie das die Daten zeigen (vgl. Tab. 1):

\begin{tabular}{|l|c|c|c|c|c|c|}
\hline & lassen $\mathrm{K}^{4}$ & lassen $\mathrm{P}$ & fare K & fare $\mathrm{P}$ & lasciare & hagy \\
\hline Agens & 26 & 18 & 53 & 6 & 54 & 47 \\
\hline Vorgangsträger & 87 & 21 & 76 & 4 & 83 & 77 \\
\hline Zustandsträger & 16 & 11 & 28 & - & 9 & 42 \\
\hline Thema/Patiens & 1 & 1 & 15 & 2 & 8 & 32 \\
\hline
\end{tabular}

$4 \quad \mathrm{~K}$ und P kennzeichnen jeweils die kausative bzw. die permissive Lesart. 


\begin{tabular}{|l|c|c|c|c|c|c|}
\hline Handlungsträger & 8 & 9 & 9 & 2 & 2 & 2 \\
\hline Besitzer & - & - & 3 & - & - & - \\
\hline unklar & 5 & 2 & 6 & - & - & - \\
\hline insgesamt & 143 & 62 & 190 & 14 & 157 & 200 \\
\hline
\end{tabular}

Tab. 1: Infinitivsubjekte bei kanonischen Kausativkonstruktionen

Bei jedem Matrixverb überwiegen Belege, in denen ich das Infinitivsubjekt als Vorgangsträger klassifiziert habe. Diese Ausdrücke bezeichnen typischerweise etwas Unbelebtes, an dem sich ein Vorgang vollzieht:

(14) DT. Das Treibhausgas lässt die Meere auch versauern. (B08/JAN.00092 Berliner Ztg., 02.01.2008 [S. 12])

Marginal kommen hier auch Ausdrücke vor, die das semantische Merkmal [+belebt] aufweisen. Das können sowohl Bezeichnungen von Personen (15) als auch Bezeichnungen von Nicht-Personen (16) sein:

(15) Dт. [... s sagte der Trainer, der sein Star-Ensemble [...] bei der Konditionsarbeit schwitzen lässt. (DPA09/JAN.01207 dpa, 04.01.2009)

(16) DT. Bisher ging man davon aus, dass ein langer Herbst die Pflanzen länger wachsen und CO2 aufnehmen lässt. (B08/JAN.00578 Berliner Ztg., 03.01.2008 [S. 15])

Agensphrasen mit den Merkmalen [+belebt] [+Handlungskontrolle] stehen entgegen der Ausgangshypothese - erst an zweiter Stelle:

(17) DT. [...] in der EU wäre es unmöglich, das Volk über etwas so Heikles abstimmen zu lassen. (A09/JAN.00322 St. Galler Tagbl., 05.01.2009, S. 23)

Am dritthäufigsten sind in jeder Vergleichssprache Infinitivsubjekte, die den Zustandsträger/Experiencer bezeichnen, die als solche typischerweise Personen (oder zumindest Lebewesen) sind:

(18) DT. [...] der Wind vom nahen See ließ die Zuschauer frieren. (DPA09/ JAN.00474 dpa, 02.01.2009) 
In jeder Vergleichssprache gab es jedoch auch Belege mit unbelebtem Zustandsträger:

(19) DT. Dort hatte mein Bekannter sein Auto am Samstagabend im Schneetreiben sicherheitshalber stehen lassen. (BRZ09/JAN.01538 Braunschw. Z., 06.01.2009)

Im Deutschen und im Italienischen waren solche Belege nur vereinzelt anzutreffen, demgegenüber haben sie im Ungarischen mehr als die Hälfte der Belege ausgemacht.

Des Weiteren gab es im Untersuchungskorpus Belege, bei denen ich das Infinitivsubjekt als Thema/Patiens klassifiziert habe:

(20) DT. Zwei englische Rettungssanitäter haben einen Mann nach Medienberichten absichtlich sterben lassen [...]. (DPA09/JAN.00082 dpa, 01.01.2009)

Hierbei zeigen die Vergleichssprachen erhebliche Unterschiede: Während im Italienischen und im Ungarischen solche Belege vergleichsweise häufig anzutreffen sind, scheinen sie im Deutschen einen Randbereich auszumachen.

Ferner gab es Belege, in denen das Infinitivsubjekt den Handlungsträger bezeichnet:

(21) Dт. Jan-Axel Alavaara ließ die Scheibe an den Außenpfosten klatschen [...]. (BRZ09/JAN.01390 Braunschw. Z., 05.01.2009)

Die meisten Belege mit diesem semantischen Merkmal gab es im deutschen Korpus und bei kausativ verwendetem fare. Bei lasciare und bei hagy waren solche Belege nur vereinzelt anzutreffen.

Schließlich gab es im Italienischen, beim Matrixverb fare, drei Belege, bei denen ich das Infinitivsubjekt als Besitzer klassifiziert habe:

$\begin{array}{llll}\text { (22) Іт. } & \text { Le } & \text { avevo } & \text { chiesto di farmi } \\ \text { ihr } & \text { hab.IMP.3SG } & \text { gebeten PRÄP machen.mir } \\ \text { avere } & l^{\prime} & \text { indirizzo [...] } & \text { (COLFIS) } \\ \text { haben } & \text { DEF.ART } & \text { Adresse } \\ & \text { Ich habe sie gebeten, mir die Adresse zu geben.' }\end{array}$


Zusammenfassend lässt sich feststellen, dass die Infinitivsubjekte in kanonischen KK typischerweise das semantische Merkmal Agens bzw. Vorgangsträger aufweisen. Infinitivsubjekte mit den Merkmalen Zustandsträger/Experiencer, Thema/Patiens sowie Handlungsträger kommen insgesamt seltener vor. Die Vergleichssprachen weisen lediglich bei den Proportionen von Thema/Patiens und Handlungsträger Unterschiede auf, die auch durch den relativ geringen Umfang der Untersuchungskorpora erklärt werden können.

\subsection{Kausativpassiv}

Wenn das KP als eine Art Diathese zu den kanonischen KK aufgefasst werden soll, dann sollten die Infinitivsubjekte in Konstruktionen mit dem KP über ähnliche semantische Eigenschaften verfügen wie die in kanonischen KK. Die Ergebnisse der Pilotanalyse zeigen, dass das nicht der Fall ist. Außerdem weist das KP auch andere Eigenschaften auf, die sich von den kanonischen KK unterscheiden: Bei den Matrixverben, die sowohl eine kausative als auch eine permissive Lesart erlauben (lassen bzw. fare), ist beim KP die permissive Lesart häufiger als in KK (vgl. Molnár 2018: 89, Molnár i. Dr.).

Darüber hinaus scheint in den Vergleichssprachen die Reflexivierung das KP zu erzwingen, da sonst die Referenzidentität zwischen Satzsubjekt und Reflexivum nicht hergestellt werden kann:

(23a) DT. Hans läßt sich $_{\mathrm{i}}$ von Fritz überreden. (Zifonun et al. 1997: 1420) (23b) DT. Hans ${ }_{\mathrm{i}}$ läßt Fritz sich $_{\mathrm{j}} / \mathrm{ihn}_{\mathrm{i}, \mathrm{k}}$ überreden. (Zifonun et al. 1997: 1420)

Belege für KP ohne Reflexivierung sind im Untersuchungskorpus mit Ausnahme von lassen $K$ und fare $K$ vergleichsweise selten (vgl. Tab. 2):

\begin{tabular}{|l|c|c|c|c|c|c|}
\hline & lassen K & lassen P & fare K & fare P & lasciare & hagy \\
\hline Reflexivierung & 50 & 108 & 84 & 47 & 193 & 161 \\
\hline gesamt & 91 & 110 & 152 & 48 & 200 & 179 \\
\hline
\end{tabular}

Tab. 2: Reflexivierung (vgl. Molnár i. Dr.) 
Somit kann angenommen werden, dass sowohl die Permissivität als auch die Reflexivierung die Semantik der Infinitivsubjekte beeinflussen kann.

Die Ergebnisse der Korpusanalyse zeigen ein differenziertes Bild (vgl. Tab. 3):

\begin{tabular}{|l|c|c|c|c|c|c|}
\hline & lassen K & lassen P & fare K & fare P & lasciare & hagy \\
\hline Agens & 79 & 48 & 138 & 26 & 59 & 81 \\
\hline Handlungsträger & 1 & 2 & - & 1 & 9 & 2 \\
\hline Verursacher & - & 46 & 1 & 17 & 73 & 44 \\
\hline Vorgangsträger & - & - & 1 & - & 4 & 11 \\
\hline Zustandsträger & - & - & - & - & - & 1 \\
\hline Besitz & - & - & - & - & 1 & - \\
\hline Vermittler & 6 & - & - & - & - & - \\
\hline Instrument & 3 & 10 & 4 & - & - & 19 \\
\hline Ort & 2 & - & - & - & - & - \\
\hline Gefühl & - & - & - & 4 & 16 & - \\
\hline lexikalisiert & - & 3 & - & - & - & - \\
\hline unklar & - & 5 & 8 & - & 38 & 19 \\
\hline insgesamt & 91 & 110 & 152 & 48 & 200 & 179 \\
\hline
\end{tabular}

Tab. 3: Infinitivsubjekte bei Kausativpassiv

Bei der kausativen Lesart der Konstruktionen mit lassen und fare überwiegen Infinitivsubjekte, die ich als Agens klassifiziert habe, d.h., Infinitivsubjekte, die belebt (oder metonymisch interpretiert) sind und auch Handlungskontrolle aufweisen:

(24) DT. Zurzeit lässt der Verwaltungsrat die Situation von externen Experten analysieren. (A09/JAN.00001 St. Galler Tagbl., 03.01.2009, S. 31)

Das ist auch die Gruppe, die die meisten Belege ohne Reflexivierung aufweist, obwohl Reflexivierung auch hier möglich ist:

(25) DT. Ausgerechnet ein Mann aus dem arabischen Emirat Katar lässt sich von ihr die Route ansagen. (BRZ09/JAN.00509 Braunschw. Z., 03.01.2009) 
Bei den Belegen mit permissiver Lesart bzw. mit permissiven Verben sind Infinitivsubjekte mit diesem semantischen Merkmal - mit Ausnahme von hagy - wesentlich seltener, wobei die meisten Belege Reflexivität aufweisen:

(26) DT. Ich bin es leid, mich von Ideologen bevormunden zu lassen. (S94/ H02.00127 Spiegel, 10.01.1994 [S. 13])

Bei der permissiven Lesart sind Belege sehr häufig, in denen das Infinitivsubjekt als Verursacher klassifiziert werden kann:

(27) DT. Auch von den Minusgraden lassen sich die Feiernden die Partylaune nicht verderben. (DPA09/JAN.00007 dpa, 01.01.2009)

Solche Infinitivsubjekte kennzeichnen etwas Unbelebtes und kommen fast ausschließlich in Belegen mit Reflexivierung vor.

Alle anderen Gruppen sind vergleichsweise schwach vertreten. Dabei ist auffallend, dass Infinitivsubjekte, die ich als Vorgangsträger klassifiziert habe und die bei kanonischen KK häufig vorkommen (vgl. Tab. 1), beim KP einen Randbereich bilden: Die meisten Belege gab es im Ungarischen (28), das deutsche Untersuchungskorpus enthält keine solchen Belege:

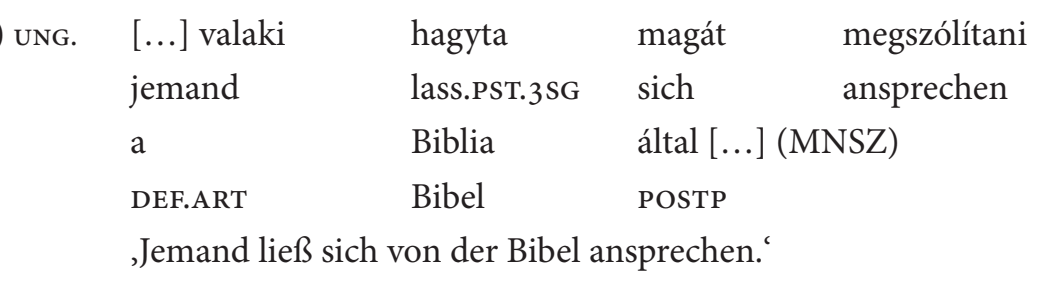

In jeder Vergleichssprache gab es Belege mit dem semantischen Merkmal „Instrument":

(29) DT. Um dem Instrument unbeschadet die Geheimnisse seiner Bauweise zu entlocken, ließen sie es kurzerhand von einem Computertomographen durchleuchten. (DPA09/APR.05559 dpa, 10.04.2009) 
Belege, die ich als „Gefühl“ klassifiziert habe, gab es nur im Italienischen (und nur mit permissiver Lesart und mit Reflexivierung):

\begin{tabular}{|c|c|c|c|}
\hline \multirow[t]{5}{*}{ (30) IT. } & Freedon Nadd & lasciò & pervadere \\
\hline & Freedon Nadd & lass.REM.3SG & durchdringen \\
\hline & dalla & rabbia [...] (PAISĀ) & \\
\hline & PRÄP.DEF & Wut & \\
\hline & $\begin{array}{l}\text {,Freedon Nadd } \\
\text { durchdringen) }\end{array}$ & de wütend' (wörtlich: l & \\
\hline
\end{tabular}

Belege mit dem Merkmal „Vermittler“ gab es hingegen nur beim kausativen lassen:

(31) DT. Den Gläubigen, [...], ließ der Bischof seine Entscheidung durch die jeweiligen Priester in den Gottesdiensten am vergangenen Wochenende mitteilen. (T09/SEP.04221 taz, 30.09.2009, S. 23)

Schließlich muss angemerkt werden, dass es hier (vor allem bei lasciare und hagy) relativ viele Belege gab, bei denen ich die semantischen Merkmale der Infinitivsubjekte nicht eindeutig bestimmen konnte.

Zusammenfassend kann festgestellt werden, dass die Infinitivsubjekte beim KP typischerweise die semantische Rolle „Agens“ bzw. „Verursacher“ haben, andere semantische Rollen sind vergleichsweise schwach vertreten. Unterschiede lassen sich weniger zwischen den einzelnen Vergleichssprachen, sondern eher zwischen der kausativen und der permissiven Lesart feststellen.

\subsection{Fazit und Konsequenzen}

Anhand der Pilotanalyse kann Folgendes festgestellt werden: Die Infinitivsubjekte in kanonischen KK sowie im KP zeigen divergierende semantische Eigenschaften. Die semantische Gruppe „Vorgangsträger“, die bei den kanonischen KK die zahlenmäßig größte Gruppe ausmacht, scheint im KP lediglich ein Randbereich zu sein. Demgegenüber ist die semantische Gruppe „Verursacher“, die im Kausativpassiv - insbesondere in permissiven Konstruktionen - relativ 
häufig ist, bei den kanonischen Kausativkonstruktionen nicht vertreten. Lediglich die semantische Rolle „Agens“ ist in beiden Typen mit vergleichsweise großer Vorkommenshäufigkeit vertreten. Somit ist das die einzige Gruppe, für die die Hypothese, Kausativpassiv sei eine Art Diathese zu kanonischen Kausativkonstruktionen, getestet werden kann. Auch hier müssen die Belege, die Reflexivität aufweisen, ausgeklammert werden, da Reflexivierung das Kausativpassiv erzwingt (s. oben). Zahlenmäßige Ergebnisse zur Reflexivierung sind aus Tab. 4 ersichtlich.

\begin{tabular}{|l|c|c|c|c|c|c|}
\hline & lassen K & lassen P & fare K & fare P & lasciare & hagy \\
\hline Reflexivierung & 46 & 47 & 78 & 25 & 52 & 73 \\
\hline keine Refl. & 33 & 1 & 60 & 1 & 7 & 8 \\
\hline
\end{tabular}

Tab. 4: Agens bei Kausativpassiv - Reflexivierung

In einem weiteren Schritt wurde untersucht, ob in den Belegen ohne Reflexivierung ein anderer Konstruktionstyp (wie die kanonische KK) möglich gewesen wäre. Die Ergebnisse werden in Tab. 5 zusammengefasst:

\begin{tabular}{|l|c|c|c|c|c|c|}
\hline & lassen K & lassen P & fare K & fare P & lasciare & hagy \\
\hline $\begin{array}{l}\text { Alternative } \\
\text { möglich }\end{array}$ & 17 & 1 & 71 & 1 & 3 & 4 \\
\hline $\begin{array}{l}\text { Alternative } \\
\text { nicht möglich }\end{array}$ & 16 & 1 & 19 & - & 4 & 4 \\
\hline
\end{tabular}

Tab. 5: Belege ohne Reflexivierung

Schließlich wurden die Belege untersucht, in denen auch ein anderer Konstruktionstyp (die kanonische KK) möglich gewesen wäre. Es wurde versucht, zu ermitteln, welche Gründe für die Wahl des KP sprechen.

Hierbei scheinen die Vergleichssprachen erhebliche Unterschiede aufzuweisen: Im Deutschen unterscheiden sich die Varianten mit kanonischen KK und die mit KP in ihrem Mitteilungswert:

(32a) DT. Zurzeit lässt der Verwaltungsrat die Situation von externen Experten analysieren. (A09/JAN.00001 St. Galler Tagbl., 03.01.2009, S. 31)

(32b) DT. Zurzeit lässt der Verwaltungsrat externe Experten die Situation analysieren. 
Bei den kanonischen KK ist nur die Reihenfolge Infinitivsubjekt - Akkusativobjekt des Infinitivs grammatisch (da es keinen morphologischen Unterschied zwischen den beiden Satzgliedern gibt). Beim KP scheint die Reihenfolge Akkusativobjekt des Infinitivs - Infinitivsubjekt die vorherrschende zu sein. Da im Deutschen die Satzglieder nach ihrem steigenden Mitteilungswert angeordnet werden, bewirkt diese Stellung eine Art Hervorhebung des Infinitivsubjekts.

Das Italienische zeigt ein anderes Bild: In dieser Sprache kann in KK das Infinitivsubjekt nicht als direktes Objekt erscheinen, wenn der Infinitiv schon ein ausgedrücktes direktes Objekt hat. In diesen Fällen erscheint das Infinitivsubjekt in der Regel als indirektes Objekt (mit der Präposition $a$ ):

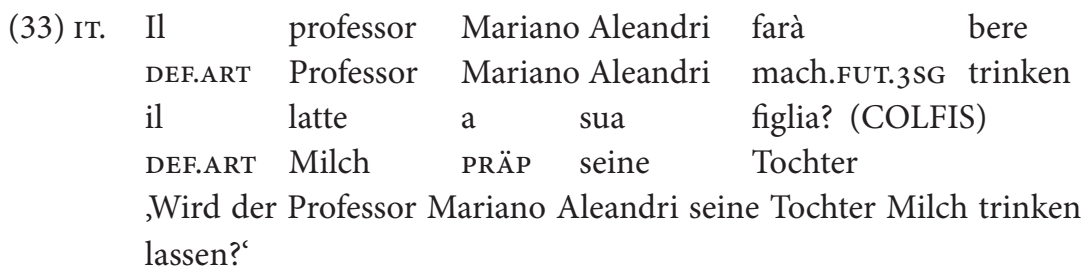

Die typische Reihenfolge der Elemente ist in solchen Fällen: Objekt des Infinitivs - logisches Subjekt des Infinitivs (vgl. auch Skytte 1983: 61). Für die Fälle mit KP ist dieselbe Reihenfolge charakteristisch: ${ }^{5}$

$\begin{array}{llllll}\text { (34) IT. } & \text { Poi } & \text { si } & \text { sono fatti } & \text { consegnare } & \text { soldi } \\ \text { dann } & \text { sich } & \text { sein.3PL } & \text { gemacht } & \text { überreichen } & \text { DEF.ART Geld.PL } \\ \text { dal } & \text { titolare } & \text { Leonida Bruni [...] } & \text { (COLFIS) } & & \\ \text { PRÄP.DEF } & \text { Besitzer } & \text { Leonida Bruni } & & \\ \text {,Dann haben sie sich vom Besitzer Leonida Bruni das Geld überreichen } \\ \text { lassen. }\end{array}$

Aus diesem Grund kann die Wahl des KP weder mit Satzgliedstellungseigenschaften noch mit der Hervorhebung des Infinitivsubjekts erklärt werden. Den beiden Konstruktionsweisen werden in der Literatur verschiedene Bedeutungs-

\footnotetext{
Diese Wortstellungseigenschaften beziehen sich auf die Fälle, in denen das direkte Objekt des Infinitivs durch eine NP ausgedrückt wird. Pronominale Objekte werden an das Matrixverb klitisiert.
} 
nuancen zugesprochen (vgl. Serianni 1991: 551), ohne dass der Unterschied erklärt würde. Skytte (1983: 57ff.) behauptet, der Infinitiv habe in den Fällen mit KP eine passivische Bedeutung. Somit könnte der Bedeutungsunterschied etwa wie folgt umschrieben werden:

(35a) Iт. Lo fece registrare dal notaio, [...] (COLFIS) das mach.REM.3Sg aufzeichnen PRÄP.DEF Notar ,Er ließ das vom Notar aufzeichnen` (d.h., er hat veranlasst, dass das vom Notar aufgezeichnet wird).

(35b) IT. Lo fece registrare al notaio das mach.REM.3SG aufzeichnen PRÄP.DEF Notar ,Er ließ den Notar das aufzeichnen' (d.h., er hat den Notar veranlasst, dass er das aufzeichnet).

Der Bedeutungsunterschied ist ziemlich subtil und lässt sich schwer erfassen, er wird aber von Muttersprachlern durchaus wahrgenommen, wie mir das von einer Informantin bestätigt wurde.

Für das Ungarische scheint das KP die Reihenfolge Akkusativobjekt des Infinitivs - Infinitivsubjekt zu ermöglichen, was mit akkusativischem oder dativischem Infinitivsubjekt nicht möglich ist. Somit verändert sich auch die Informationsstruktur des Satzes.

\section{Zusammenfassung und Ausblick}

Die Pilotanalyse hat gezeigt, dass das Kausativpassiv unter den Vergleichssprachen nur im Italienischen als eine mögliche Diathese zu kanonischen Kausativkonstruktionen aufgefasst werden kann. Diese Funktion ist aber auch im Italienischen eingeschränkt, da das Kausativpassiv in mehreren Fällen (wie z. B. bei Reflexivierung) den einzig möglichen Konstruktionstyp darstellt.

Im Deutschen und im Ungarischen scheint in den Fällen, in denen auch eine alternative Konstruktion möglich ist, das Kausativpassiv eher zur informationstrukturellen Gliederung der Sätze beizutragen.

Um mehr über das Funktionieren des Kausativpassivs erfahren zu können, sind weitere Korpusanalysen notwendig, wobei Belege ohne Reflexivierung erfasst werden sollten. 


\section{Literatur}

\subsection{Korpora}

COLFIS: http://www.ge.ilc.cnr.it/corpus.php; (gesichtet am 15.02.2019).

http://ge.ilc.cnr.it/corpus_lem.php (gesichtet am 15.02.2019).

DeReKo: https://cosmas2.ids-mannheim.de/cosmas2-web (gesichtet am 15.02. 2019).

MNSZ: http://corpus.nytud.hu/mnsz (gesichtet am 15.02.2019).

PAISÀ: http://www.corpusitaliano.it (gesichtet am 15.02.2019).

\subsection{Sekundärliteratur}

Dowty, David (1991): Thematic Proto-Roles and Argument Selection. In: Language 67/3, 547-619.

Duden (2009): Duden. Die Grammatik. 8. Aufl. Mannheim u.a.: Dudenverlag.

Gunkel, Lutz (2003): Infinitheit, Passiv und Kausativkonstruktionen im Deutschen. Tübingen: Stauffenburg (Studien zur deutschen Grammatik 67).

Foley, William / van Valin, Robert Jr. (1984): Functional Syntax and Universal Grammar. Cambridge: Cambridge University Press (Cambridge Studies in Linguistics 38).

Hyvärinen, Irma (1989): Zu finnischen und deutschen verbabhängigen Infinitiven. Eine valenztheoretische kontrastive Analyse. Frankfurt a. M.: Lang.

Molnár, Krisztina (2018): Infinitivkonstruktionen als Objekte im Sprachvergleich. Berlin u.a.: Lang (Szegediner Schriften zur gemrnaistischen Linguistik 8).

Molnár, Krisztina (i. Dr.): Kausativkonstruktionen und Kausativpassiv im Deutschen und im Sprachvergleich.

Primus, Beatrice (2012): Semantische Rollen. Heidelberg: Winter (KEGLI 12). Serianni, Luca (1991): Grammatica italiana. Italiano comune e lingua letteraria. Torino: UTET.

Skytte, Gunver (1983): La sintassi dell'infinito in italiano moderno. Vol. I-II. Kopenhagen: Munksgaard.

Zifonun, Gisela / Hoffmann, Ludger / Strecker, Bruno (1997): Grammatik der deutschen Sprache. Berlin / New York: de Gruyter (Schriften des IDS 7). 